\title{
Optical to ultraviolet spectra of sandwiches of benzene and transition metal atoms: Time dependent density functional theory and many-body calculations
}

\author{
Martinez, Jose Ignacio; García Lastra, Juan Maria; Lopez, M. J.; Alonso, J. A.
}

Published in:

Journal of Chemical Physics

Link to article, DOI:

$10.1063 / 1.3300129$

Publication date:

2010

Document Version

Publisher's PDF, also known as Version of record

Link back to DTU Orbit

Citation (APA):

Martinez, J. I., García Lastra, J. M., Lopez, M. J., \& Alonso, J. A. (2010). Optical to ultraviolet spectra of sandwiches of benzene and transition metal atoms: Time dependent density functional theory and many-body calculations. Journal of Chemical Physics, 132(4), 044314. https://doi.org/10.1063/1.3300129

\section{General rights}

Copyright and moral rights for the publications made accessible in the public portal are retained by the authors and/or other copyright owners and it is a condition of accessing publications that users recognise and abide by the legal requirements associated with these rights.

- Users may download and print one copy of any publication from the public portal for the purpose of private study or research.

- You may not further distribute the material or use it for any profit-making activity or commercial gain

- You may freely distribute the URL identifying the publication in the public portal 


\title{
Optical to ultraviolet spectra of sandwiches of benzene and transition metal atoms: Time dependent density functional theory and many-body calculations
}

\author{
J. I. Martínez, ${ }^{1,2,3, a)}$ J. M. García-Lastra, ${ }^{3,4,5}$ M. J. López, ${ }^{1}$ and J. A. Alonso ${ }^{1,4,6}$ \\ ${ }^{1}$ Departamento de Física Teorica, Atomica y Optica, Universidad de Valladolid, 47011 Valladolid, Spain \\ ${ }^{2}$ Departamento de Física Teórica de Materia Condensada, Universidad Autónoma de Madrid, \\ E-28047 Madrid, Spain \\ ${ }^{3}$ Department of Physics, Center for Atomic-Scale Materials Design, Technical University of Denmark, \\ DK-2800 Lyngby, Denmark \\ ${ }_{5}^{4}$ Departamento de Física de Materiales, Universidad del Pais Vasco, 20018 San Sebastian, Spain \\ ${ }^{5}$ Nano-Bio Spectroscopy Group and European Theoretical Spectroscopy Facility (ETSF), \\ 20018 San Sebastian, Spain \\ ${ }^{6}$ Donostia International Physics Center, 20018 San Sebastian, Spain
}

(Received 16 September 2009; accepted 7 January 2010; published online 28 January 2010)

\begin{abstract}
The optical spectra of sandwich clusters formed by transition metal atoms (titanium, vanadium, and chromium) intercalated between parallel benzene molecules have been studied by time-dependent density functional theory (TDDFT) and many-body perturbation theory. Sandwiches with different number of layers, including infinite chains, are considered. The lowest excitation energy peaks in the spectra are characteristic of the robust bonding in these complexes. The excitation energies vary in a systematic way with the metal atoms and with the cluster size, and so these materials could be used to tune the optical properties according to specific functionality targets. The differences in the spectra could be used to identify relative abundances of isomers with different spins in experimental studies. As a salient feature, this theoretical spectroscopic analysis predicts the metallization of the infinite $(\mathrm{TiBz})_{\infty}$ chain, which is not the case of $(\mathrm{CrBz})_{\infty}$. () 2010 American Institute of Physics. [doi:10.1063/1.3300129]
\end{abstract}

\section{INTRODUCTION}

The interest in the bonding between transition metal atoms and benzene began with the observation that certain colored solutions obtained by the addition of metal salts to liquid benzene arise from metal-benzene complexes. ${ }^{1,2}$ The interest on these complexes grew up soon after species, such as dibenzene chromium $\left(\mathrm{C}_{6} \mathrm{H}_{6}\right)_{2} \mathrm{Cr}$ (Ref. 3), were synthesized and found to be very stable. In recent years, the investigation of sandwiches and other complexes of metal atoms has progressed because of their relevance to catalysis, magnetic and optical materials, polymers, spintronic devices, molecular sensors, and other applications (see, e.g., Refs. 4-17).

Despite of the difficulty of synthesizing these materials, the introduction of laser vaporization techniques in pulsed nozzle supersonic expansion sources, combined with mass spectrometry detection, ${ }^{18}$ has made possible studies of their bonding energetics, spectroscopic properties, and structures. Kurikawa et $a .^{19}{ }^{19}$ detected clusters containing transition metal atoms $(\mathrm{M})$ and benzene molecules $(\mathrm{Bz})$ with strong preference for species with $\left[\mathrm{M}_{n} \mathrm{Bz}_{n+1}\right]^{+}$stoichiometries. The neutral analogs have been recently studied by magnetic deflection. ${ }^{8}$ The stoichiometries of these clusters led to the proposal of a structure of sandwiches with the $M$ atoms intercalated between benzene units. The magnetism observed

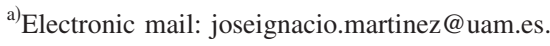

in some of them was associated to the spin-coupling along the axis of the sandwich. Calculations for small $\mathrm{M}_{n} \mathrm{Bz}_{n+1}$ clusters $(n \leq 6)$ and for infinite $(\mathrm{MBz})_{\infty}$ chains confirm the linear sandwich structure of these complexes as the most stable one. ${ }^{20}$ Recently, a theoretical study of the electronic and magnetic properties for $(\mathrm{MBz})_{\infty}$ polymers with $\mathrm{M}=\mathrm{Sc}$, $\mathrm{Ti}, \mathrm{V}, \mathrm{Cr}$, and $\mathrm{Mn}$ has shown that all chains, except $(\mathrm{CrBz})_{\infty}$, are metallic. ${ }^{21}$

Optical and UV spectroscopies are powerful tools for obtaining information on the electronic and atomic structure of clusters. Since the structure of a cluster is generally not directly accessible experimentally, ${ }^{22}$ its characterization can be done with a combination of optical spectroscopy and theoretical calculations. ${ }^{23}$ Optical absorption spectroscopy experiments for gas phase clusters containing transition metal atoms are quite challenging. Experiments and calculations of direct absorption or laser induced fluorescence are needed in the case of $\mathrm{M}_{n} \mathrm{Bz}_{n+1}$ clusters. The possibility of varying the number of layers in the sandwich and the nature of the intercalated atoms raises expectations of interesting optical properties and possible applications of these materials.

We present first-principles calculations of the photoabsorption cross sections of $\mathrm{M}_{n} \mathrm{Bz}_{n+1}$ sandwiches with $\mathrm{M}=\mathrm{Ti}$, $\mathrm{V}, \mathrm{Cr}$, and $n \leq 3$, and for infinite $(\mathrm{TiBz})_{\infty}$ and $(\mathrm{CrBz})_{\infty}$ chains. The choice of $\mathrm{Ti}, \mathrm{V}$, and $\mathrm{Cr}$ is based on: (i) the experimental evidence that the tendency to form multidecker sandwiches is stronger for the early than for the late transition metals; ${ }^{19}$ (ii) other metal atoms, like silver or copper, have virtually no 
low-lying electronic states that are coupled to the groundstate through allowed optical transitions; ${ }^{24}$ and (iii) the possibility of comparing the absorption properties of magnetic $\left(\mathrm{V}_{n} \mathrm{Bz}_{n+1}\right)$ and nonmagnetic $\left(\mathrm{Ti}_{n} \mathrm{Bz}_{n+1}\right.$ and $\left.\mathrm{Cr}_{n} \mathrm{Bz}_{n+1}\right)$ systems. ${ }^{8,10}$ Our aim is to study the changes in the electronic properties, as reflected in the photoabsorption spectrum, as the sandwich size and the metallic element are varied. We find that the optical properties of the sandwiches can be tuned by varying the number of units. In addition, we find a metallization effect in the infinite chains, confirming the prediction of Xiang et al. ${ }^{21}$

\section{CALCULATION OF THE PHOTOABSORPTION SPECTRUM}

A method extensively used to calculate the photoabsorption spectrum of clusters is the time-dependent density functional theory (TDDFT). ${ }^{23,25}$ The implementations used in practice have some limitations due to the approximate description of exchange and correlation effects between the electrons, like the known underestimation of the magnitude of the energy gap in semiconductors. A similar error could be expected for the gap between occupied and unoccupied states (HOMO-LUMO gap) in finite clusters. Possible errors in the gap and in the asymptotic behavior of the exchangecorrelation $(\mathrm{XC})$ potential in a finite cluster could affect the energies of the excited electronic states and thus the photoabsorption spectrum. For this reason, the TDDFT results are compared here with accurate results obtained from manybody perturbation theory.

We have first performed full optimizations of the geometries of these clusters, starting with the structures proposed in the literature and considering different spin-multiplicity states. DFT, implemented in the OCTOPUS package, ${ }^{26}$ was used with the PBE0 hybrid XC functional, ${ }^{27}$ which mixes a generalized gradient (GGA) functional with a predetermined amount (25 per cent) of exact exchange. The PBE0 functional improves the description of band gaps compared to uncorrected GGA functionals, through the incorporation of a fraction of Hartree-Fock exchange, ${ }^{28,29}$ and this provides a better starting point for TDDFT calculations. Semicore corrected norm-conserving pseudopotentials ${ }^{30,31}$ were employed. In agreement with previous work, ${ }^{9-11,19,20}$ our calculations show that the most stable structures of these complexes are sandwiches with the metal atoms intercalated and positioned in the axis of the sandwich (z-axis), as seen in Fig. 1. As previously reported for the $\mathrm{V}, \mathrm{Nb}$, and $\mathrm{Ta}$ systems, ${ }^{10,20}$ small rotation angles $\left(1-3^{\circ}\right)$ of the benzene rings relative to each other around the $\mathbf{z}$-axis are found depending on the spin state. In the benzene molecules at the two ends of the sandwich, the planes of $\mathrm{C}$ and $\mathrm{H}$ hexagons are slightly shifted relative to each other along the $\mathbf{z}$-axis by $\sim 0.05-0.10 \AA$. The infinite $(\mathrm{MBz})_{\infty}$ chains have been modeled as having pure $\mathrm{D}_{6 \mathrm{~h}}$ symmetry. The ground states of the $\mathrm{Ti}_{n} \mathrm{Bz}_{n+1}$ and $\mathrm{Cr}_{n} \mathrm{Bz}_{n+1}$ structures are nonmagnetic. The $\mathrm{V}_{n} \mathrm{Bz}_{n+1}$ clusters are more complex. The configuration with spin magnetic moment $\mu_{z}=0 \mu_{B}$ in which the two $\mathrm{V}$ atoms are antiferromagnetically coupled is very close in energy to a ferromagnetic configuration with $\mu_{z}=2 \mu_{B}$ in $\mathrm{V}_{2} \mathrm{Bz}_{3}$. The

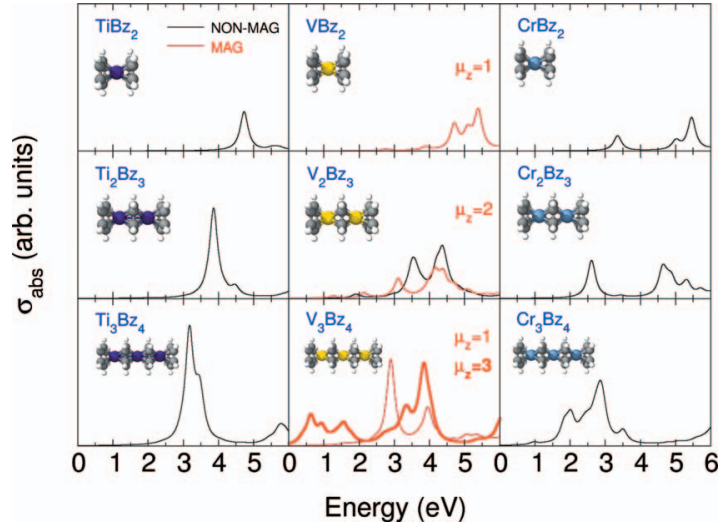

FIG. 1. Calculated TDDFT photoabsorption cross sections (in arbitrary units) for $\mathrm{M}_{n} \mathrm{Bz}_{n+1}$ sandwich clusters, with $n \leq 3$ and $\mathrm{M}=\mathrm{Ti}, \mathrm{V}$, and $\mathrm{Cr}$, for excitation energies up to $6 \mathrm{eV}$. Spectra for two nearly degenerate states with different net spin magnetic moments are shown for $\mathrm{V}_{2} \mathrm{Bz}_{3}$ and $\mathrm{V}_{3} \mathrm{Bz}_{4}$. The excitation peaks have been broadened by Lorentzian functions. The geometrical structures are included as insets.

truly nonspin-polarized state is substantially above in energy $(0.35 \mathrm{eV})$ with respect to the other two. In the case of $\mathrm{V}_{3} \mathrm{Bz}_{4}$, the magnetic configurations with $\mu_{z}=1 \mu_{B}$ and $\mu_{z}=3 \mu_{B}$ have very similar energies. For $\mathrm{VBz}_{2}$, the $\mu_{z}=1 \mu_{B}$ ground-state is clearly preferred.

Then, the photoabsorption spectrum is obtained using the TDDFT with the formalism developed by Casida. ${ }^{32}$ The ingredients for this calculation are the electronic singleparticle energies and orbitals of the ground state described above and a XC kernel, for which the self-interaction corrected (SIC) adiabatic local density approximation (ALDA) $)^{33}$ is employed. The spectra of $\mathrm{M}_{n} \mathrm{Bz}_{n+1}$ sandwiches with $n$ $\leq 3$ are shown in Fig. 1 for energies up to $6 \mathrm{eV}$. In the three series $(\mathrm{M}=\mathrm{Ti}, \mathrm{V}, \mathrm{Cr})$ the absorption energy thresholds decrease as more $\mathrm{MBz}$ units are added to the structure: the absorption threshold occurs near 4.5, 3.5, and $2.5 \mathrm{eV}$ for the growing $\mathrm{Ti}_{n} \mathrm{Bz}_{n+1}$ sandwiches, and near 3.0, 2.0, and $1.5 \mathrm{eV}$ for the growing $\mathrm{Cr}_{n} \mathrm{Bz}_{n+1}$ systems. In the more complex $\mathrm{V}_{n} \mathrm{Bz}_{n+1}$ structures, the red shift is also observed. However, two different spin states are nearly degenerate for $\mathrm{V}_{2} \mathrm{Bz}_{3}$ and the same occurs for $\mathrm{V}_{3} \mathrm{Bz}_{4}$, and the absorption threshold and the form of the spectrum are very sensitive to the spin state. It is likely that an experimental spectrum will be a superposition of the two spectra for each of those clusters. A general property is that the absorption intensity becomes higher as the number of layers in the sandwich increases.

The spectra of the $\mathrm{Ti}_{n} \mathrm{Bz}_{n+1}$ sandwiches are characterized by a main peak centered at 4.7, 3.9, and $3.2 \mathrm{eV}$ for $n=1,2$, and 3 , respectively. This peak corresponds to transitions from $3 \mathrm{~d}$ states of $\mathrm{Ti}$ to benzene states. The intensity of the peak scales with the number of $\mathrm{TiBz}$ units forming the cluster. Two absorption regions can be distinguished in the spectra of $\mathrm{CrBz}_{2}$ and $\mathrm{Cr}_{2} \mathrm{Bz}_{3}$ : a low energy peak centered at 3.3 and $2.6 \mathrm{eV}$, respectively, followed by a broader absorption region at higher energies. Both spectra are similar except for a relative shift of about $0.6 \mathrm{eV}$. The origin of the low energy peaks is the same as in the clusters with Ti. The high energy region of the spectra arises from internal transitions in the metal atoms, from $3 \mathrm{p}$ states to the open $3 \mathrm{~d}$ shell. The spectrum of 
$\mathrm{Cr}_{3} \mathrm{Bz}_{4}$ differs from the other two. The overlap between the atomic $3 \mathrm{~d}$ orbitals along the axis of the cluster lowers the energy of the atomic transitions and the second peak approaches closely to the first one. The spectra of $\mathrm{V}_{n} \mathrm{Bz}_{n+1}$ show some common features with those of the clusters containing $\mathrm{Ti}$ and $\mathrm{Cr}$, that is, a shift of the spectrum to lower energies as $n$ increases. Of course, the analysis becomes more complex because different spin configurations appear for different $n$. An extended low-energy absorption region between 0 and $2 \mathrm{eV}$ appears for the state of $\mathrm{V}_{3} \mathrm{Bz}_{4}$ with $\mu_{z}$ $=3 \mu_{B}$. This arises from the increased occupation of orbitals of the spin up channel, giving rise to low energy transitions. The $3 p-3 d$ transitions discussed above for the $\mathrm{Cr}$ sandwiches occur at energies above $6 \mathrm{eV}$ in the $\mathrm{Ti}$ and $\mathrm{V}$ sandwiches. The same $3 \mathrm{p}-3 \mathrm{~d}$ transitions occur in the isolated $\mathrm{Ti}, \mathrm{V}$ and $\mathrm{Cr}$ atoms at energies about $4.5-5 \mathrm{eV}$.

To explore the influence of quasiparticle and electronhole interaction effects in the optical spectra of the sandwiches we have used the GW (Green function-screened interaction) approach and solved the Bethe-Salpeter equation (BSE), respectively. ${ }^{34}$ For this purpose: (i) as a starting point, taking the optimized geometries, the ground state electronic structure of $\mathrm{Ti}_{n} \mathrm{Bz}_{n+1}$ and $\mathrm{Cr}_{n} \mathrm{Bz}_{n+1}$ was obtained with the PWSCF $\operatorname{code}^{35}$ using an LDA XC-functional ${ }^{33}$ and normconserving pseudopotentials; ${ }^{30}$ (ii) electronic quasiparticle corrections were calculated within the $\mathrm{GW}$ approximation to the XC self-energy; ${ }^{36}$ (iii) finally, the BSE was solved for coupled electron-hole excitations, ${ }^{37}$ thereby accounting for the screened electron-hole attraction and the unscreened electron-hole exchange. Steps (ii) and (iii) were performed with the YAMBO code. ${ }^{38}$ In addition, infinite $(\mathrm{TiBz})_{\infty}$ and $(\mathrm{CrBz})_{\infty}$ chains have also been studied.

This formalism includes excitonic effects, which are more important in strongly correlated systems, and is able to distinguish the response to each individual light polarization component. In Fig. 2, a comparison between photoabsorption spectra calculated by the TDDFT and GW-BSE formalisms is shown. The GW-BSE spectra include separately the $\mathbf{x}, \mathbf{y}$, and $\mathbf{z}$ polarization contributions and the averaged spectra. For $\mathrm{TiBz}_{2}$ and $\mathrm{Ti}_{2} \mathrm{Bz}_{3}$, the spectra obtained by both formalisms show the same single-peak structure, but the excitonic corrections shift the peaks to lower energies, by 0.75 and $1.2 \mathrm{eV}$, respectively. This excitonic shift does not occur in $\mathrm{Ti}_{3} \mathrm{Bz}_{4}$. In this system, with more $\mathrm{TiBz}$ units, the overlap between the d-type orbitals along the axis of the sandwich induces their dispersion, reducing the electronic correlation and the excitonic contribution. This overlap leads to the metallization of the $(\mathrm{TiBz})_{\infty}$ chain. For the $\mathrm{Cr}_{n} \mathrm{Bz}_{n+1}$ sandwiches, the excitonic effect increases as the number of $\mathrm{CrBz}$ units increases. A low electronic correlation can be noticed for the $\mathrm{CrBz}_{2}$, for which the TDDFT and GW-BSE spectra are similar. In this case, the TDDFT description of the excitations is accurate. As the number of units in the $\mathrm{CrBz}$ sandwiches increases, the excitonic effects become dominant. The reductions in the excitation energies of the first peak with respect to the TDDFT values are $0.1,0.6$, and $0.8 \mathrm{eV}$ for $n=1,2$, and 3 , respectively. The infinite $(\mathrm{CrBz})_{\infty}$ chain exhibits an intense low energy excitation peak centered at $0.5 \mathrm{eV}$. The peaks of the TDDFT spectra of $\mathrm{CrBz}_{2}$ and $\mathrm{Cr}_{2} \mathrm{Bz}_{3}$ occurring at high

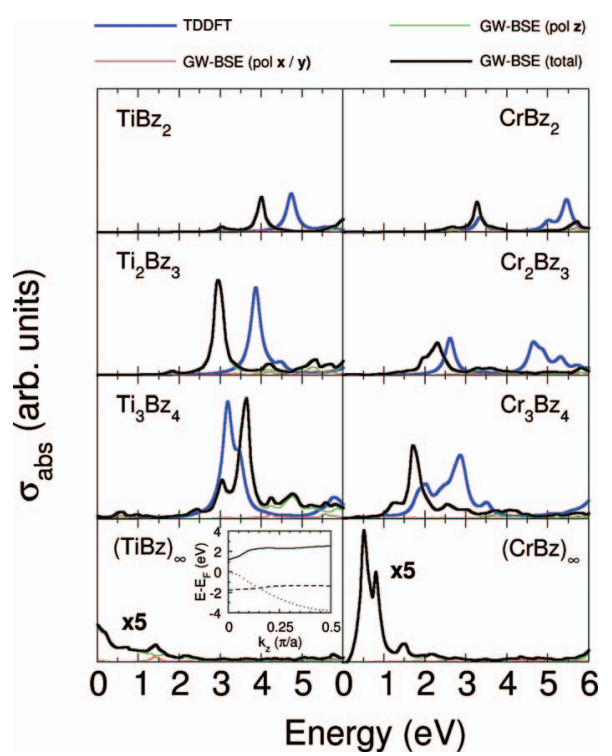

FIG. 2. Calculated GW-BSE photoabsorption cross sections (in arbitrary units) of $\mathrm{Ti}_{n} \mathrm{Bz}_{n+1}$ and $\mathrm{Cr}_{n} \mathrm{Bz}_{n+1}$ sandwich clusters $(n \leq 3)$ and infinite chains. The individual $\mathbf{x}, \mathbf{y}$, and $\mathbf{z}$ polarization contributions and the spectrum averaged over the three polarizations are shown in different colors. TDDFT results are included for comparison. The inset in the bottom-left panel shows the GW energy band diagram for the infinite TiBz chain: $d_{x z}$,

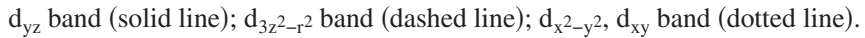

excitation energies are not present in the GW-BSE spectra shown in Fig. 2. The reason is that those excitations are shifted to even higher energies. The pronounced sizedependent behavior of the optical spectrum, in which the visible absorption frequencies decrease with cluster size and saturate for the chain may possibly be used in tailoring the optical properties.

In Fig. 3, the TDDFT photoabsorption cross sections for $\mathrm{TiBz}_{2}$ and $\mathrm{CrBz}_{2}$, calculated by using two different $\mathrm{XC}$ functionals, the LDA of Perdew and Zunger $(\mathrm{PZ})^{33}$ and the GGA-PBE0, ${ }^{27}$ with the same LDA+SIC kernel, are compared to the GW-BSE cross sections. The agreement with the GW-BSE spectrum is better for the TDDFT results obtained using the PBE0 hybrid XC functional, showing the main excitation peaks much closer to the GW-BSE profile than those calculated with the local PZ parametrization. This is mainly due to the improved description of the HOMOLUMO gap given by a GGA functional over the LDA. Although combining a GGA ground-state description with an LDA kernel is apparently not entirely consistent, this is com-

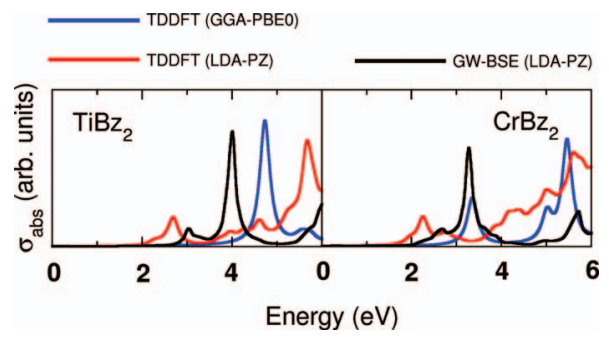

FIG. 3. Comparison between the TDDFT photoabsorption cross sections for the $\mathrm{TiBz}_{2}$ and $\mathrm{CrBz}_{2}$ sandwich clusters, calculated by using two different $\mathrm{XC}$ functionals (LDA-PZ and GGA-PBE0), and the GW-BSE cross sections. The excitation peaks have been broadened by Lorentzian functions. The geometrical structures are shown as insets in Fig. 1. 


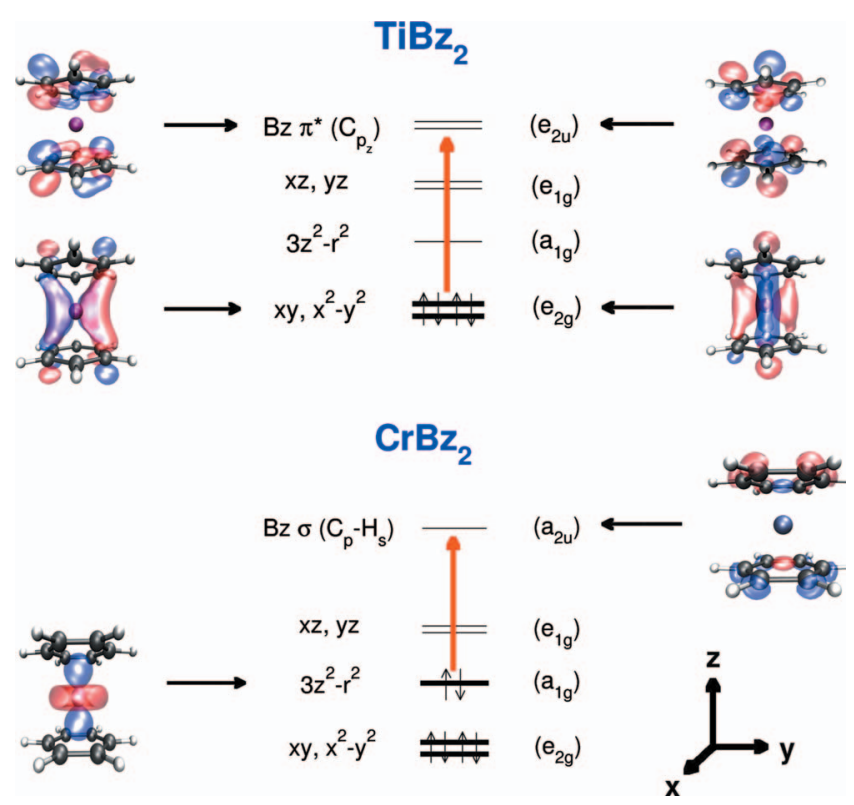

FIG. 4. Frontier orbitals of $\mathrm{TiBz}_{2}$ (top panel) and $\mathrm{CrBz}_{2}$ (bottom panel), and axis set chosen in the calculations. On the right hand side, the orbitals are labeled in accordance with the $\mathrm{D}_{6 \mathrm{~h}}$ point group symmetry. The thick vertical red arrows indicate the transitions responsible for the low energy peaks of Fig. 2.

mon practice. ${ }^{39}$ The reason is that the use of a better kernel does not affect much the results as long as the single-particle orbitals have been calculated with a good static $\mathrm{v}_{x c}(\mathbf{r})$ potential, like the PBE0 hybrid functional.

Figure 4 illustrates the electronic transitions responsible for the low energy peaks in the spectra. In the case of $\mathrm{TiBz}_{2}$, the lowest energy excitation peak corresponds to a transition from the $d_{x y}, d_{x^{2}-y^{2}}$ orbitals of Ti to a LUMO-like benzene doublet orbital. These orbitals are labeled respectively as $e_{2 g}$ and $e_{2 u}$ in $D_{6 h}$ symmetry, and thus the transition is only allowed through $\mathbf{z}$-axis polarized light (with $\mathrm{a}_{2 \mathrm{u}}$ symmetry), while it is forbidden through $\mathbf{x}$ and $\mathbf{y}$-axis polarized light $\left(\mathrm{e}_{1 \mathrm{u}}\right.$ symmetry). This fact is confirmed by the GW-BSE photoabsorption results, in which the $\mathbf{z}$-axis polarization gives the only contribution to the lowest energy peak of Fig. 2. Moreover, it can be checked that there are no other allowed transition between the $\mathrm{TiBz}_{2} \mathrm{HOMO}$ and the frontier orbitals, apart from the mentioned $\mathrm{e}_{2 \mathrm{~g}} \rightarrow \mathrm{e}_{2 \mathrm{u}}$ transition. In the case of $\mathrm{CrBz}_{2}$ the lowest energy peak corresponds to a transition from the $\mathrm{d}_{3 \mathrm{z}^{2}-\mathrm{r}^{2}}$ state of $\mathrm{Cr}$ (with $\mathrm{a}_{1 \mathrm{~g}}$ symmetry) to a benzene orbital with $a_{2 \mathrm{u}}$ symmetry, corresponding to a $\sigma$-like bond formed by hydrogen s orbitals and carbon p orbitals. The transition is only allowed through $\mathbf{z}$-axis polarized light and it is the only transition allowed between the $\mathrm{CrBz}_{2} \mathrm{HOMO}$ and the frontier orbitals. This analysis can be readily extended to other sandwich sizes, because the nature of the transitions in those $\mathrm{M}_{n} \mathrm{Bz}_{n+1}$ clusters (with $n>1$ and $\mathrm{M}=\mathrm{Ti}$ and $\mathrm{Cr}$ ) is the same than in the simplest ones with $n=1$.

Due to the orbital overlap in the cartesian $\mathbf{z}$ direction, the bands corresponding to the $\mathrm{d}_{\mathrm{xy}}, \mathrm{d}_{\mathrm{x}^{2}-\mathrm{y}^{2}}$ doublet and the $\mathrm{d}_{3 \mathrm{z}^{2}-\mathrm{r}^{2}}$ orbitals of the intercalated transition metal atoms broaden as $n$ increases, inducing a red shift in the lower energy peaks. As expected, the dispersion is more pronounced for the $d_{x y}$, $d_{x^{2}-y^{2}}$ doublet than for the $d_{3 z^{2}-r^{2}}$ orbital, because the orbitals

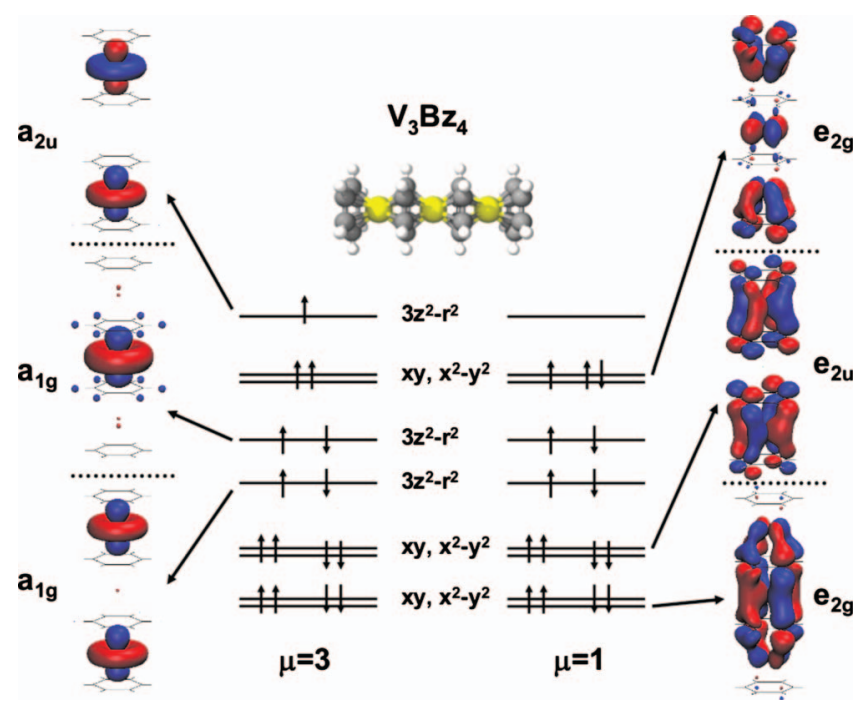

FIG. 5. Frontier orbitals of $\mathrm{V}_{3} \mathrm{Bz}_{4}$ for its two different spin configurations with $\mu=1 \mu_{B}$ and $\mu=3 \mu_{B}$. On the left and right hand sides, the orbitals are labeled in accordance with the $\mathrm{D}_{6 \mathrm{~h}}$ point group symmetry.

of the doublet show a stronger overlap with the benzene orbitals (see Fig. 4). A salient feature of the infinite chain is that the $\mathrm{d}_{3 \mathrm{z}^{2}-\mathrm{r}^{2}}$ band crosses the $\mathrm{d}_{\mathrm{xy}}, \mathrm{d}_{\mathrm{x}^{2}-\mathrm{y}^{2}}$ band (see inset in Fig. 2). In the $(\mathrm{CrBz})_{\infty}$ chain, both the $d_{3 z^{2}-r^{2}}$ and the $d_{x y}$, $\mathrm{d}_{\mathrm{x}^{2}-\mathrm{y}^{2}}$ bands are filled, and the dispersion only gives rise to an intense redshift of the low energy absorption peak with respect to the $\mathrm{CrBz}_{2}$ spectrum (see Fig. 2). In contrast, the $\mathrm{d}_{3 \mathrm{z}^{2}-\mathrm{r}^{2}}$ orbital is empty in $\mathrm{TiBz}_{2}$ and thus the dispersion leads to the metallization of the $(\mathrm{TiBz})_{\infty}$. This fact has been already reported in a recent work by Xiang et al., ${ }^{21}$ who used the PW91 functional ${ }^{40}$ in their work. However, it is well known that DFT tends to underestimate the gaps, predicting a metallic behavior for small gap semiconductors. This trend is corrected by the $\mathrm{GW}$ calculations, ${ }^{41}$ so we propose the metallization of the $(\mathrm{TiBz})_{\infty}$ chain predicted by Xiang et al. as a reliable effect, with possible interest in applications involving tunable electro-optical properties in nanoscale devices. The present results also predict that the $(\mathrm{CrBz})_{\infty}$ chain does not become metallic, again in agreement with Xiang et al. $^{21}$

It is worthwhile to apply a similar orbital-based analysis to $\mathrm{V}_{3} \mathrm{Bz}_{4}$ in its two different spin configurations, in order to shed light on the source of the differences seen in the lowenergy region of the spectra for the magnetic states with $\mu=1 \mu_{B}$ and $\mu=3 \mu_{B}$. The ordering of the electronic levels and the form of the associated orbitals are depicted in Fig. 5. The low energy peaks in the photoabsorption spectrum of the state with $\mu=3 \mu_{B}$ arise from excitations from its HOMO orbital. This molecular orbital corresponds to an antisymmetric linear combination of the $\mathrm{d}_{3 \mathrm{r}^{2}-\mathrm{z}^{2}}$ orbitals of the two $\mathrm{V}$ atoms located at the extremes of the sandwich. It is remarkable that this is the only one of the last four frontier orbitals with ungerade symmetry. Low energy transitions are allowed from this level, but not from the HOMO-1, HOMO-2, or HOMO-3 levels. On the other hand, that orbital is empty in the case of $\mu=1 \mu_{B}$; therefore, there will be no transitions from this orbital when $\mu=1 \mu_{B}$.

Some photoemission experiments have been performed 
on the neutral and anionic species of several of these sandwiches. Recently, Sohnlein et al. ${ }^{42}$ have performed ZEKEPES measurements on bis-benzene complexes of titanium and vanadium, which provide information on the energetics of the occupied orbitals, but not of the unoccupied ones, and hence they cannot be compared with the optical spectra. On the other hand, Zheng et al. $^{43}$ have performed anion photoelectron measurements on $\left(\mathrm{Ti}_{n} \mathrm{Bz}_{m}\right)^{-}$. These experiments provide information on the energy of the LUMO of the neutral $\mathrm{Ti}_{n} \mathrm{Bz}_{m}$ system with respect to its occupied orbitals. It can be inferred from that work than the LUMO of $\mathrm{TiBz}_{2}$ is about $1 \mathrm{eV}$ above the occupied states, in good agreement with our calculations in which the HOMO-LUMO gap is $1.13 \mathrm{eV}$. However, this energy is much lower than the one obtained for the first intense peak in the photoabsorption spectrum, namely $4 \mathrm{eV}$ in the GW-BSE calculations. This is due to the fact that all the transitions from the occupied frontier orbitals of $\mathrm{TiBz}_{2}$ to its LUMO are forbidden by symmetry. The same feature occurs for the rest of the $\mathrm{Ti}_{n} \mathrm{Bz}_{n+1}$ systems studied in this work.

\section{CONCLUSION}

A suitable combination of TDDFT and GW-BSE calculations has allowed an exhaustive analysis of the optical spectra of sandwich clusters formed by transition metal atoms intercalated between parallel benzene molecules. The low excitation energy peaks are characteristic of the robust bonding in this kind of complexes, and different metal atoms and cluster sizes could be used to tune the optical properties according to specific functionality targets. The differences in the spectra can be used to identify relative abundances of isomers with different spins in experimental studies. The present results are in good agreement with the experiments and calculations found in recent literature.

\section{ACKNOWLEDGMENTS}

This work was supported by MICINN of Spain (Grant No. MAT2008-06483-C03-01) and Junta de Castilla y León (Grant Nos. VA017A08 and GR23). J.A.A. acknowledges an Ikerbasque fellowship from the Basque Foundation for Science, and the hospitality of DIPC. J.M.G-L. acknowledges funding from Spanish MICINN through Juan de la Cierva and José Castillejo programs.

\footnotetext{
${ }^{1}$ S. Winstein and H. J. Lucas, J. Am. Chem. Soc. 60, 836 (1938).

${ }^{2}$ R. S. Mulliken, J. Am. Chem. Soc. 74, 811 (1952).

${ }^{3}$ E. O. Fischer and W. Z. Hafner, Z. Naturforsch. B 10, 665 (1955).

${ }^{4}$ N. J. Long, Metallocenes (Blackwell Sciences, Oxford, 1998).

${ }^{5}$ T. D. Jaeger, D. van Heijnsbergen, S. J. Klippenstein, G. von Helden, G. Meijer, and M. A. Duncan, J. Am. Chem. Soc. 126, 10981 (2004).

${ }^{6}$ K. Miyajima, A. Nakajima, S. Yabushita, M. B. Knickelbein, and K. Kaya, J. Am. Chem. Soc. 126, 13202 (2004).

${ }^{7}$ A. Naitabdi, J. P. Bucher, P. Gerbier, P. Rabu, and M. Drillon, Adv. Mater. 17, 1612 (2005)
}

${ }^{8}$ K. Miyajima, S. Yabushita, M. B. Knickelbein, and A. Nakajima, J. Am. Chem. Soc. 129, 8473 (2007).

${ }^{9}$ M. A. Duncan, Int. J. Mass Spectrom. 272, 99 (2008).

${ }^{10}$ J. Wang, P. H. Acioli, and J. Jellinek, J. Am. Chem. Soc. 127, 2812 (2005); 106, 3208 (2006).

${ }^{11}$ J. Wang and J. Jellinek, J. Phys. Chem. A 109, 10180 (2005).

${ }^{12}$ T. Yasuike, A. Nakajima, S. Yabushita, and K. Kaya, J. Phys. Chem. A 101, 5360 (1997).

${ }^{13}$ K. Miyajima, K. Muraoka, M. Hashimoto, T. Yasuike, S. Yabushita, A. Nakajima, and K. Kaya, J. Phys. Chem. A 106, 10777 (2002).

${ }^{14}$ P. Weis, P. R. Kemper, and M. T. Bowers, J. Phys. Chem. A 101, 8207 (1997).

${ }^{15}$ R. Pandey, B. K. Rao, P. Jena, and M. A. Blanco, J. Am. Chem. Soc. 123, 7744 (2001)

${ }^{16}$ A. K. Kandalam, B. K. Rao, P. Jena, and R. Pandey, J. Chem. Phys. 120, 10414 (2004).

${ }^{17}$ J. Kua and K. M. Tomlin, J. Phys. Chem. A 110, 11988 (2006).

${ }^{18}$ T. G. Dietz, M. A. Duncan, D. E. Powers, and R. E. Smalley, J. Chem. Phys. 74, 6511 (1981).

${ }^{19}$ T. Kurikawa, H. Takeda, M. Hirano, K. Judai, T. Arita, S. Nagao, A. Nakajima, and K. Kaya, Organometallics 18, 1430 (1999).

${ }^{20}$ Y. Mokrousov, N. Atodiresei, G. Bihlmayer, and S. Blügel, Int. J. Quantum Chem. 106, 3208 (2006).

${ }^{21}$ H. Xiang, J. Yang, J. G. Hou, and Q. Zhu, J. Am. Chem. Soc. 128, 2310 (2006).

${ }^{22}$ J. A. Alonso, Structure and Properties of Atomic Nanoclusters (Imperial College Press, London, 2005).

${ }^{23}$ J. I. Martínez, A. Castro, A. Rubio, and J. A. Alonso, J. Comput. Theor. Nanosci. 3, 761 (2006).

${ }^{24}$ K. F. Willey, P. Y. Cheng, M. B. Bishop, and M. A. Duncan, J. Am. Chem. Soc. 113, 4721 (1991); K. F. Willey, C. S. Yeh, D. L. Robbins, and M. A. Duncan, J. Phys. Chem. 96, 9106 (1992).

${ }^{25}$ M. A. L. Marques and E. K. U. Gross, Annu. Rev. Phys. Chem. 55, 427 (2004)

${ }^{26}$ M. A. L. Marques, A. Castro, G. F. Bertsch, and A. Rubio, Comput. Phys. Commun. 151, 60 (2003).

${ }^{27}$ C. Adamo, G. E. Scuseria, and V. Barone, J. Chem. Phys. 111, 2889 (1999).

${ }^{28}$ A. Alkauskas and A. Pasquarello, Physica B 401-402, 670 (2007).

${ }^{29}$ X. Wu, E. J. Walter, A. M. Rappe, R. Car, and A. Selloni, Phys. Rev. B 80, 115201 (2009).

${ }^{30}$ N. Troullier and J. L. Martins, Phys. Rev. B 43, 1993 (1991).

${ }^{31}$ C. Hartwigsen, S. Goedecker, and J. Hutter, Phys. Rev. B 58, 3641 (1998).

${ }^{32}$ M. E. Casida, in Recent Advances in Density Functional Methods, Part I, edited by D. P. Chong (World Scientific, Singapore, 1995), p. 155.

${ }^{33}$ J. P. Perdew and A. Zunger, Phys. Rev. B 23, 5048 (1981).

${ }^{34}$ G. Onida, L. Reining, and A. Rubio, Rev. Mod. Phys. 74, 601 (2002).

${ }^{35}$ S. Baroni, A. D. Corso, S. de Gironcoli, and P. Giannozzi QUANTUMESPRESSO package, 2005 (http://www.quantum-espresso.org/).

${ }^{36}$ M. S. Hybertsen and S. G. Louie, Phys. Rev. B 34, 5390 (1986).

${ }^{37}$ S. Albrecht, L. Reining, R. Del Sole, and G. Onida, Phys. Rev. Lett. 80, 4510 (1998); L. X. Benedict, E. L. Shirley, and R. B. Bohn, ibid. 80, 4514 (1998); M. Rohlfing and S. G. Louie, ibid. 83, 856 (1999).

${ }^{38}$ A. Marini, C. Hogan, M. Grüning, and D. Varsano, Comput. Phys. Commun. 180, 1392 (2009).

${ }^{39}$ J. I. Martínez and J. A. Alonso, Phys. Rev. B 76, 205409 (2007).

${ }^{40}$ J. P. Perdew, J. A. Chevary, S. H. Vosko, K. A. Jackson, M. A. Pederson, D. J. Sing, and C. Fiolhais, Phys. Rev. B 46, 6671 (1992).

${ }^{41}$ M. van Schilfgaarde, T. Kotani, and S. Faleev, Phys. Rev. Lett. 96, 226402 (2006).

${ }^{42}$ B. R. Sohnlein, Y. X. Lei, and D. S. Yang, J. Chem. Phys. 127, 114302 (2007).

${ }^{43}$ W. J. Zheng, J. M. Nilles, O. C. Thomas, and K. H. Bowen, Chem. Phys. Lett. 401, 266 (2005). 